\title{
Kernos
}

Revue internationale et pluridisciplinaire de religion grecque antique

6 | 1993

Varia

\section{L'histoire de Méléagre : plaidoyer pour une analyse de l'ensemble du mythe}

Jean-Michel Renaud

\section{(2) OpenEdition \\ Journals}

Édition électronique

URL : http://journals.openedition.org/kernos/553

DOI : $10.4000 /$ kernos.553

ISSN : 2034-7871

Éditeur

Centre international d'étude de la religion grecque antique

Édition imprimée

Date de publication : 1 janvier 1993

Pagination : 291-300

ISSN : 0776-3824

Référence électronique

Jean-Michel Renaud, «L'histoire de Méléagre : plaidoyer pour une analyse de l'ensemble du mythe », Kernos [En ligne], 6| 1993, mis en ligne le 07 avril 2011, consulté le 01 mai 2019. URL : http:// journals.openedition.org/kernos/553; DOI : 10.4000/kernos.553 


\section{L'HISTOIRE DE MÉLÉAGRE :}

\section{PLAIDOYER POUR UNE ANALYSE DE LENSEMBLE DU MYTHE}

Depuis quelques années, les études sur les mythes, et particulièrement les mythes grecs, se sont multipliées ${ }^{1}$. Pendant un certain temps, les spécialistes avaient hésité à faire l'exégèse des mythes ${ }^{2}$. L'abus des explications naturalistes chères à l'école de Max Müller les avait rendus particulièrement méfiants. Heureusement, de nouvelles écoles ont repris l'analyse des mythes sur une base plus large et souvent mieux assurée. On sait le rôle de pionnier que Marie Delcourt a joué dans ces nouveaux développements. L'intérêt témoigné actuellement pour l'étude des mythes et la multiplication des travaux qui leur sont consacrés n'implique aucunement qu'un accord général existe entre spécialistes sur des points qui semblent pourtant essentiels. Ainsi, d'âpres discussions continuent d'avoir lieu sur la nature du mythe ${ }^{3}$, même si l'on reconnaît qu'il n'est pas définissable. Les différents systèmes d'exégèse sont critiqués et, parfois, on a le sentiment que la notion de mythe a été tellement étendue qu'elle tend à se diluer ${ }^{4}$.

Le présent article ne vise aucunement à ajouter au nombre déjà considérable des publications qui, tantôt claires, tantôt alourdies de jargon, débattent des questions théoriques relatives à l'être, à l'origine et au devenir du mythe. Ce qu'on voudrait simplement mettre ici en valeur, c'est une méthode d'approche du mythe qui rende compte du plus

1 Notamment en France à la suite des travaux de l'École de Jean-Pierre Vernant et de Marcel Detienne.

2 On en trouvera un exemple parmi d'autres dans l'ouvrage au demeurant bien documenté de J. Davreux, La légende de la prophéteresse Cassandre, LiègeParis, 1942 (Bibliothèque de la Faculté de Philosophie et Lettres, 94).

3 L'acception de $\mu \nu \tilde{\theta} 0$ s chez les Anciens semble avoir été différente de ce qu'elle est chez les Modernes. Cf. C. CALAM E, «Mythe» et «rite» en Grèce : des catégories indigènes?, in Kernos, 4 (1991), p. 179-204, et J. Boulogne, Le mythe pour les Anciens Grecs, in Uranie, 1 (1991), p. 17-30. - Chez les Modernes, cf. D. Dubuisson, La mythologie au XXe s., in Uranie, 1 (1991), p. 159-168, et A. DEREMETZ, Histoire des définitions du mythe, in Mythe et création. Actes du Coll. Intern. d'Arras 1992, à paraître.

4 Cf. M. Detienne, L'invention de la mythologie, Paris, 1981. 
grand nombre possible de ses virtualités. Parmi les multiples études sur le sujet, beaucoup décèlent, dans des récits mythiques, des traits communs, tantôt à l'intérieur d'une même culture, tantôt dans des ensembles plus vastes. Parmi les traits communs, on a souligné très fréquemment ces derniers temps la présence et l'importance d'éléments initiatiques et de rites de passage ${ }^{5}$. Il est indéniable que de tels traits existent et qu'ils sont très nombreux : initiation de l'adolescent ou de l'adolescente qui atteint l'âge adulte, initiation de groupes ou d'individus isolés qui désirent adhérer à une société fermée, ou encore d'individus qui cherchent à acquérir un pouvoir ou un savoir extraordinaires ${ }^{6}$. Toute vie humaine peut même être présentée comme une vaste épreuve initiatique qui prépare le passage dans l'Au-Delà. C'est dire que la matière est vaste et que pratiquement tous les récits mythiques comportent des traits initiatiques. Faut-il se borner à mentionner ceuxci sans aller plus loin, comme on le voit dans un certain nombre d'ouvrages, et ne risque-t-on pas de fausser à nouveau le système d'explication en attirant l'attention uniquement sur un aspect du problème, important sans doute, mais qui n'est pas le seul ${ }^{7}$ ? Un des caractères du mythe que pratiquement chacun reconnaît est son aspect global. Le récit mythique, comme la poésie, envisage la réalité dans son ensemble, comme un tout, et non dans ses différents éléments constitutifs. C'est ce qui rend le mythe à la fois impossible à définir et difficile à analyser. Pour s'en tenir à la Grèce antique, on sait combien les mythes y sont multiples et divers et on ne peut les ramener à une seule notion, fût-elle universellement répandue. Chaque mythe est donc riche de toute une série d'informations et d'enseignements qui reflètent les réponses aux questions que s'est posée la société grecque à différentes époques. L'analyse d'un mythe déterminé devrait s'efforcer non seulement de déceler tel ou tel trait isolé, mais aussi de saisir, dans l'ensemble du récit, le plus possible de réponses données. Il s'agit là d'une tentative qui n'aboutira sans doute jamais totalement dans la mesure où les récits mythiques sont par nature chargés de sens divers que l'on ne peut avoir la prétention d'exprimer tous.

5 Citons par exemple Les rites d'initiation. Actes du Coll. de Liège et Louvain-laNeuve 20-21 nov. 1984, Louvain-la-Neuve, 1986 (Homo Religiosus, 13), et L'initiation. Actes du Coll. Intern. de Montpellier 11-14 avril 1991, Montpellier, Université Paul Valéry, 1992, 2 vol.

6 Cf. P. W ATHELET, Synthèse du Colloque, in L'initiation, op. cit. , 2, p. 261-271.

7 Cf. C. CAlame, Prairies intouchées et jardins d'Aphrodite : espaces «initiatiques» en Grèce, in L'initiation, op. cit., 2, p. 103-118. 
Concrètement, pour tenter l'exégèse d'un mythe, on réunira toutes les versions que l'Antiquité a laissées du mythe en question, non seulement dans la littérature, mais aussi dans la documentation iconographique. On saisira les différents enseignements du mythe à travers la trame du récit en tenant compte de ses variantes, plus ou moins nombreuses, qui sont toutes chargées de signification et dont la comparaison est riche d'informations.

On examinera le sens éventuel du nom des personnages, la signification des attitudes ou des objets décrits dans le récit en les rapprochant de cas analogues qui apparaissent dans d'autres mythes. On s'attachera aux liens que les héros peuvent avoir avec des divinités, à ce qu'on sait par diverses sources des attributions de ces divinités, et aux recoupements qui peuvent se présenter entre les mythes et les faits de culte $^{8}$. Tout être humain, comme toute société, est amené à s'interroger sur un certain nombre de problèmes. Certains de ces problèmes intéressent une société à toutes les époques, voire l'humanité tout entière. D'où vient le monde ? D'où vient l'homme ? Pourquoi l'homme meurt-il ? Quels sont les rôles respectifs de l'homme et de la femme ? Autant de questions qui ont toujours dû être posées. D'autres questions peuvent avoir un intérêt beaucoup plus limité. Pourquoi faut-il ou ne faut-il pas manger tel aliment dans telle circonstance ? D'où vient telle cérémonie cultuelle ? etc. Telles sont les questions, générales ou particulières. On peut concevoir que, dans certaines cultures, elles n'aient pas toutes reçu une réponse sous la forme d'un récit mythique. Même sur les questions les plus générales, les réponses apportées sont différentes d'une société à l'autre, et elles peuvent varier à l'intérieur d'une société, selon les époques.

En ce qui concerne la Grèce, surgit une difficulté d'ordre chronologique. La société de l'époque d'Homère et d'Hésiode est différente de ce qu'on sait, par exemple, de celle des cités grecques du Ve siècle ou de celle des grandes monarchies hellénistiques. Diverses considérations permettent toutefois de limiter l'importance de ces différences. Depuis les premiers témoignages littéraires jusqu'à la fin du Ve siècle et malgré les différences évidentes dont il faudra tenir compte, la société grecque semble avoir fait preuve d'une remarquable continuité. Dans l'ensemble, les mythes grecs renvoient à l'époque archaïque. Ils ont été

8 Pour la méthode, cf. P. WATHELET, Dictionnaire des Troyens de l'Iliade, Liège, Faculté de Philosophie et Lettres, 1988 (Documenta et Instrumenta, 1), p. 113133. 
repris et actualisés par les grands tragiques grecs, et spécialement par Euripide. Les époques postérieures, alexandrine et romaine, ont vu des changements profonds dans la société, mais elles sont marquées aussi, dans le domaine des Lettres, par un souci de continuer et de sauver la tradition classique. Les mythes grecs ont sans doute alors perdu une partie de leur valeur paradigmatique - les mythes tendent à devenir mythologie-, mais des auteurs même très tardifs veillent à continuer ou à sauver des traditions plus anciennes. Dès le IVe siècle, et plus encore à l'époque alexandrine et au-delà, se constitue une littérature d'antiquaires qui collectionne les récits mythologiques et les particularités cultuelles héritées des périodes archaïque et classique. Certains auteurs latins, dont particulièrement Virgile, participent à ce courant. L'auteur de l'Énéide possède une connaissance et un sens remarquables des mythes grecs, mais on ne peut ignorer que certains de ces mythes sont interprétés par lui dans une nouvelle perspective, celle de la grandeur de Rome. On ne peut pas ne pas en tenir compte. Faut-il encore souligner que, depuis l'époque classique, une partie du rôle des récits mythiques avait été reprise par la philosophie ? Une autre considération pratique amène à prendre en compte les témoignages d'époques diverses et très souvent tardifs. On ne possède qu'une part très réduite de la littérature antique. Particulièrement pour les périodes les plus anciennes, les sources comportent d'énormes lacunes. Ce sont souvent des auteurs, des compilateurs ou des scholiastes très tardifs qui apportent le contenu d'ouvrages disparus et qui permettent d'avoir une vue un peu plus complète des récits mythiques tels qu'ils devaient exister dans les poèmes épiques, lyriques et tragiques. La documentation est trop réduite dans les périodes les plus anciennes pour qu'on puisse avoir la prétention de dire que tel trait qui figure dans un mythe est apparu pour la première fois chez tel auteur à telle époque. Dans l'immense majorité des cas, la première attestation d'un trait ne correspond pas nécessairement à son apparition à l'intérieur du mythe.

Dans un travail récent, on s'est efforcé de faire une analyse aussi complète que possible du mythe de Méléagre ${ }^{9}$. Le mythe présente un double intérêt : il est attesté dès l'Iliade (B 642 et I 529-299) et il comporte, dans des versions différentes, des variantes inconciliables. Bien que,

9 Cf. J.-M. Renaud, Le mythe de Méléagre. Essais d'interprétation, Liège, chez l'auteur, $37 \mathrm{r}$. Naimette, B - 4000 Liège, 1993. On trouvera dans cet ouvrage les justifications et les références sur lesquelles s'appuient les divers traits de l'analyse du mythe qui seront mentionnés après son résumé. 
l'Iliade mise à part, l'ensemble du récit ne soit attesté que par des auteurs tardifs, les allusions ponctuelles qui y sont faites et les fragments que nous avons d'œuvres archaïques ou classiques montrent que le mythe a été très tôt largement connu. Sans entrer dans tous ses détails, on en donnera ici un récit linéaire, en en mentionnant toutefois les principales variantes ${ }^{10}$.

Oineus était roi de Calydon ${ }^{11}$. Il avait épousé Althaia et, de leur union, naquit notamment Méléagre. La naissance de celui-ci est marquée par des faits merveilleux ${ }^{12}$. Ainsi, les Moires se manifestent, annoncent que le héros aura des qualités exceptionnelles, mais la dernière précise que sa vie est liée à un tison enflammé et qu'elle se terminera quand le tison sera consumé. À cette nouvelle, Althaia retire le tison du foyer et l'enferme dans un coffre afin qu'il soit protégé. Plus tard, alors que Méléagre est devenu un jeune homme et qu'il a participé avec éclat à l'expédition des Argonautes, son père Oineus omet de faire un sacrifice d'offrande à Artémis. Irritée, la déesse se venge en lançant sur Calydon un sanglier aussi extraordinaire que redoutable. Le sanglier fait d'énormes ravages et Méléagre réunit une troupe de chasseurs qui regroupe tous les héros de la Grèce. Parmi ceux-ci se trouve une femme, Atalante, originaire d'Arcadie. La chasse au sanglier est engagée. Il y a quelques victimes parmi les chasseurs et, finalement, le fauve est blessé par Atalante et tué par Méléagre. Celui-ci reçoit le trophée, c'est-à-dire la hure et la peau du monstre, mais il veut en faire cadeau à Atalante dont il s'est épris. Ce cadeau suscite la réprobation des chasseurs, qui avaient déjà témoigné quelque irritation contre la présence d'une femme parmi eux. C'est spécialement le cas des oncles maternels du héros. Il se dispute avec eux, une lutte survient et Méléagre tue ses oncles maternels. Irritée de la mort de ses frères, Althaia veut les venger. Elle sort le tison du coffre et le jette au feu, provoquant du même coup la mort du héros. Dans une autre version du

10 Un abrégé de l'ensemble du mythe est indispensable à la compréhension des quelques éléments de conclusion qui seront exposés ici, mais on a conscience que cette manière de faire est théoriquement discutable, dans la mesure où elle semble favoriser une version au détriment des autres.

11 On trouvera la liste des références aux auteurs qui mentionnent le mythe dans ses différentes versions dans l'étude mentionnée à la note 9 .

12 Une autre version raconte qu'Althaia a mis au monde un rameau d'olivier en même temps que Méléagre. Cf. M. Detienne, L'écriture d'Orphée, Paris, 1989, p. 71-84. On ne peut traiter cette variante ici, mais elle est absorbée dans J.-M. RENAUD, op. cit. 
mythe, qui apparait dès l'Iliade, il n'est fait mention ni du tison, ni de l'intervention d'Atalante. Après la mort du sanglier, et à l'instigation d'Artémis, une dispute éclate entre Méléagre et ses oncles maternels. Alliés aux Courètes, ils viennent assiéger Calydon. Méléagre en tue quelques-uns. Althaia lance alors une imprécation contre son fils et, furieux de l'attitude de sa mère, celui-ci s'enferme auprès de sa femme, Cléopatrè. Malgré les supplications des habitants de Calydon, il refuse de participer au combat. Ce n'est qu'au moment où l'appartement dans lequel il s'est enfermé est attaqué et sur les instances de Cléopatrè qu'il revient au combat. Il s'y fait tuer, dans certaines versions, par une flèche d'Apollon. À la nouvelle de sa mort, Althaia et Cléopatrè se pendent et ses sœurs, sauf Déjanire et Gorgè, sont transformées en pintades. Aux Enfers, Héraclès rencontrera l'ombre de Méléagre. Il sera ému par le récit de sa vie et Méléagre lui suggérera d'épouser sa sœur Déjanire.

Comme on le voit, le mythe est complexe et le résumé qui vient d'en être donné est loin de mentionner toutes ses variantes. Le récit comporte incontestablement des thèmes initiatiques. Ainsi, la victoire du héros sur le monstre, en l'occurrence le sanglier de Calydon, peut constituer une épreuve initiatique, dont on possède d'autres exemples, comme celui du séjour d'Ulysse chez son grand-père maternel Autolycos ( $T$ 395-466). Les oncles maternels de Méléagre, dont le rôle est capital dans l'histoire, apparaissent comme des maîtres d'initiation ${ }^{13}$, de même que les Courètes, parfois présentés comme une peuplade. Leur rôle de maîtres d'initiation était déjà apparu dans un épisode antérieur à la chasse au sanglier, l'expédition des Argonautes : Méléagre, dont la grande jeunesse est soulignée en cette occasion, y est accompagné d'un de ses oncles maternels, Iphiclos, qui exerce sur lui une sorte de surveillance. En attribuant la dépouille du sanglier à Atalante et en se disputant avec ses oncles, Méléagre rate son épreuve initiatique et cet échec a des conséquences dramatiques pour lui-même et pour les siens.

Cependant, le mythe est riche d'autres enseignements, qui sont d'une grande importance pour une meilleure compréhension de la mentalité grecque. Un des personnages essentiels de tout le récit est le père de Méléagre, Oineus. C'est sa négligence qui provoque la colère d'Artémis et l'irruption du sanglier. Or, Oineus, le «Vigneron», est très

13 Cf. J. B REMMER, The Importance of the Maternal Uncle and Grandfather in Archaic and Classical Greece and Early Byzantium, in ZPE, 50 (1983), p. 173186. - Discussion détaillée de la question dans J.-M. RENAUD, op. cit. 
proche de Dionysos, qui est effectivement associé au roi dans certaines variantes du récit. Le mythe de Méléagre apparaît donc comme une opposition entre Dionysos, représenté par Oineus, et Artémis, représentée non seulement par le sanglier, mais également par Atalante et même par Méléagre, qui est donné comme un chasseur particulièrement valeureux. L'importance de Dionysos dans les mythes de l'épopée homérique n'a guère été décelée jusqu'à présent, dans la mesure où les spécialistes ont longtemps cru que Dionysos était un dieu d'origine étrangère, tardivement introduit dans le panthéon grec. Depuis le déchiffrement du linéaire $B$, et plus encore depuis la découverte récente du théonyme dans un contexte religieux sur une tablette de la Canée en Crète, on sait que Dionysos figurait déjà dans le panthéon grec à l'époque mycénienne. Le caractère itinérant du dieu et sa sauvagerie, qui avaient fait croire à une origine extérieure tardive, apparaissent à présent comme des éléments constitutifs de sa personnalité. Comme l'Iliade l'indique déjà, Dionysos est le dieu fou ${ }^{14}$, il prend le contre-pied de toutes les institutions et de tous les usages, quels qu'ils soient, il incarne le côté "irrationnel» qui existe dans tout être humain, et surtout dans tout groupe social. Or, Oineus représente bien le facteur de désordre qu'incarne Dionysos. Roi de Calydon, il omet de faire un sacrifice important et, par son attitude, il attire un malheur qui frappe tout son peuple. Dans la majorité des versions, ce n'est pas lui qui prend la décision de combattre le sanglier, mais il en laisse la responsabilité à son fils. Lors de la lutte entre les Courètes et les Calydoniens, Oineus supplie en vain Méléagre de retourner au combat. La mission d'un roi n'est pas de supplier, mais de donner des ordres, ce dont Oineus est bien incapable. Mauvais roi, il est aussi mauvais chef de famille, dans la mesure où c'est sa femme, Althaia, qui prend les décisions.

L'opposition entre Dionysos et Artémis tient à leur nature respective : Artémis est à la fois la déesse de la féminité, qui conduit la jeune fille au mariage et à la procréation, et la déesse de la nature sauvage, qui vit dans les forêts, avec ses nymphes, et qui chasse les fauves. C'est aussi elle qui défend la limite entre le monde sauvage et le monde civilisé. Contrairement aux apparences, ces deux fonctions, de gardienne du monde sauvage et de protectrice de la féminité, se rejoignent. La mentalité grecque archaïque oppose en effet la cité, qui est le monde "raisonnable" des hommes d'âge mûr et des vieillards, et

14 À ce sujet, voir P. WAthelet, Dionysos chez Homère ou la folie divine, in Kernos, 4 (1991), p. 61-82. 
la forêt, le monde sauvage, qui est le domaine de la femme. Le monde sauvage est celui qui produit toutes les forces principales à la vie, mais ces forces risquent d'être désordonnées si elles ne sont régies par la sagesse politique $(\sigma \omega \phi \rho \circ \sigma u ́ v \eta)$ des hommes. En somme, les deux éléments, la cité et le monde sauvage, sont complémentaires et aucun des deux ne peut vivre sans l'autre. Il est symptomatique que l'opposition se retrouvera à la fois dans le vocabulaire et dans la philosophie, notamment chez Aristote, qui oppose $i \lambda_{\eta}$, le bois en tant que forêt et que matière brute, et $\mu \circ \rho \phi \eta$, l'élément qui donne sa forme à la matière. Gardienne de la féminité, Artémis défend les limites qui séparent le monde sauvage du monde civilisé et de la cité. Or, Dionysos est, comme on l'a dit, le dieu qui prend le contre-pied de tous les usages. Il est donc fatal qu'un conflit éclate entre les deux divinités, dans la mesure où Dionysos ne respecte pas les limites et introduit la sauvagerie dans la cité elle-même. L'attitude d'Oineus provoque Artémis et, en représailles, celle-ci provoque l'irruption du sanglier, c'est-à-dire du monde sauvage dans le monde civilisé, puisque le sanglier détruit les cultures, les arbres, et qu'il s'en prend même aux hommes. La vengeance de la déesse ira encore plus loin, puisqu'elle provoquera une guerre entre les Courètes et les Calydoniens. Le désordre est à son comble.

En négligeant Artémis, Dionysos inflige à la déesse un affront que lui-même ne peut supporter. Chaque fois qu'une communauté a ignoré le dieu, il s'est vengé en suscitant un désordre violent. Le roi de Thèbes, Penthée, n'a pas reconnu sa divinité. Dionysos déchaîne la folie sur les femmes de Thèbes et sur l'ensemble de la cité. Dans le cas de Calydon, c'est Oineus-Dionysos qui, dans sa folie, a négligé Artémis. Celle-ci se venge avec les armes mêmes du dieu, elle abolit la limite entre le monde sauvage et le monde civilisé et elle pousse l'explosion du désordre à un point extrême. Ce désordre atteindra toute la famille d'Oineus, mais ce dernier sera le seul à survivre, fidèle à son caractère dionysiaque. Après la disparition de Méléagre, de ses frères, d'Althaia et des Méléagrides, Oineus se remariera et aura une nouvelle descendance, sans qu'il cesse pour autant d'être marqué par le désordre. L'opposition entre les deux divinités, dont témoigne le mythe de Méléagre, se retrouve dans des éléments du culte, ainsi qu'il apparaît notamment dans le rituel d'Artémis Laphria à Patras. On en trouve des attestations dans d'autres récits mythiques (mythes d'Orion, d'Ariane, légende du renard de Teumessos, etc.) ou dans d'autres cultes, particulièrement dans la Grèce du Nord. 
Le désordre introduit à Calydon par l'attitude d'Oineus aura d'autres effets. Alors que le roi néglige ou est incapable d'exercer son pouvoir, sa femme Althaia apparaît comme une femme de tête : elle tend à assumer la direction des opérations, ce qui constitue une tâche masculine. Proche par certains côtés de la Terre-Mère, elle évoque aussi Médée, notamment lorsqu'elle lance contre Méléagre une imprécation solennelle, ainsi qu'il est raconté dans l'Iliade. Althaia domine Méléagre, qui ne pourra s'affranchir de la tutelle de sa mère. Dans la scène du tison, c'est Althaia qui enferme celui-ci dans un coffre qu'elle garde en sa possession, comme si elle gardait Méléagre enfermé. Plus tard, c'est elle encore qui voudra venger la mort de ses frères ${ }^{15}$ et qui, toujours dans la version du tison, provoquera la fin du héros. Dans l'autre version, elle lance contre son fils une imprécation que l'Érinye entendra. Toujours dans la perspective d'une domination féminine, Méléagre sera fasciné par une autre femme qui est sortie de son rôle, Atalante, la chasseresse qui, comme un homme, a lutté contre Pélée lors des jeux funèbres en l'honneur de Pélias. La présence d'Atalante, une femme, parmi les chasseurs, provoque déjà des réactions d'hostilité de certains participants. Cette hostilité atteindra son comble quand Méléagre voudra accorder le trophée à Atalante pour s'assurer son amour. Troisième femme dominatrice qui intervient auprès du héros, sa femme Cléopatrè. Elle apparaît incidemment dans la version homérique, alors qu'ailleurs, Méléagre s'éprend d'Atalante. Homère, qui ne mentionne pas l'archère arcadienne, montre Méléagre irrité contre Althaia à la suite de l'imprécation que celle-ci a lancée contre lui. Le héros s'enferme dans sa chambre auprès de sa femme et refuse d'encore participer au combat contre les Courètes, malgré les nombreuses supplications de toute sa famille et des Calydoniens. Dans la version du tison, ce dernier se trouvait enfermé par Althaia dans un coffre; dans la version homérique, Méléagre est enfermé dans sa chambre au pouvoir de sa femme. Les deux situations sont parallèles. L'Iliade rappelle rapidement la généalogie de Cléopatrè et il en ressort que la mère de l'héroïne, Marpessa, était, elle aussi, une femme dominatrice. Lorsque l'assaut des Courètes se fera par trop menaçant, c'est Cléopatrè qui interviendra auprès de Méléagre et qui obtiendra de lui ce que tous les autres n'avaient pu obtenir, son retour au combat.

15 Sur le rôle du feu dans cette version du mythe, cf. J. B REMmer, La plasticité $d u$ mythe: Méléagre dans la poésie homérique, in C. CALAme (éd.), Métamorphoses du mythe en Grèce antique, Genève, 1988, p. 47. Une analyse critique de son opinion est donnée dans J.-M. RENAUD, op. cit. 
En somme, le mythe de Méléagre est l'histoire d'une initiation manquée : un héros très brillant n'a pu réussir sa vie, a raté l'épreuve initiatique, non par manque de courage ou de force, mais parce qu'il a été incapable de se libérer de l'emprise féminine. Contrairement à l'idéal de l'époque, le héros s'est laissé dominer par les femmes, et ce désordre s'inscrit dans un cadre plus large, qui est celui de l'opposition entre Dionysos et Artémis. L'échec de Méléagre tient au fait que les limites entre le monde de l'homme et le monde de la femme n'ont pas été respectées. L'action de Dionysos a brouillé les structures sociales, dont Artémis est la gardienne en ce qui concerne la femme.

Comme on le voit, le mythe de Méléagre est riche d'enseignements divers. Pour le comprendre, il faut tenir compte de ses nombreuses variantes, attestées à des époques différentes, mais qui nous apportent des éléments essentiels. Dans l'état actuel de notre documentation, nous ne pouvons fixer la date d'apparition de ces éléments. Tout au plus pouvons-nous penser que certains traits ont été développés dans un contexte déterminé. Ainsi par exemple, la version de l'Iliade donne au conflit entre Courètes et Étoliens la dimension d'une guerre, afin que, dans le discours de Phénix, ce conflit puisse être mis sur le même pied que la guerre de Troie.

Pour comprendre les mythes grecs, il convient donc de ne pas se borner à y déceler l'un ou l'autre trait isolé, mais il faut faire une analyse en profondeur des différentes versions du récit.

Rue Naimette, 37

Jean-Michel RENAUD

B - 4000 LIÈGE 